\author{
M.G. Miguel \\ E. Marques \\ R. Dias \\ S.M. Mel'nikov \\ A. Khan \\ B. Lindman
}

\title{
Associating polymer-surfactant systems
}

Invited lecture, 12th Conference of the European Colloid and Interface Society, Dubrovnik - Cavtat, 1998

M.G. Miguel ${ }^{1} \cdot$ E. Marques ${ }^{1} \cdot$ R. Dias ${ }^{1}$

S.M. Mel'nikov · A. Khan

B. Lindman $(\bowtie)$

Physical Chemistry 1

Center for Chemistry

and Chemical Engineering

P.O. Box 124, S-22100 Lund, Sweden

Permanent address:

${ }^{1}$ Departamento de Química

Universidade de Coimbra

P-3049 Coimbra, Portugal

\begin{abstract}
Some recent illustrations of the phase behavior of polymeramphiphile systems in solution are presented. Surfactant-polymer association is demonstrated for various amphiphilic synthetic and biological polymers both on a macroscopic and on a single molecular level.
\end{abstract}

Key words Polymer-surfactant systems - Association and segregation Phase behavior - Surfactant selfassembly - Catanionic vesicles

\section{Introduction}

Mixed systems of macromolecules and amphiphiles are ubiquitous in technical products (polymer-surfactant mixtures) and have a very broad role in biology (biopolymer-lipid mixtures) [1, 2]. Controlling the interactions between macromolecules and low-molecular-weight amphiphiles is the key to technical performance and biological function [3].

Depending on the combination of a macromolecule and an amphiphile, the interaction varies and, as a consequence, the macroscopic behavior. Thus various types of polymers behave quite differently, i.e., charged polymers versus nonionic, cationic versus anionic, homopolymers versus graft or comb copolymers, etc. Early investigations focused on water-soluble homopolymers interacting with simple surfactants, but in recent years much interest has concerned macromolecules with pronounced amphiphilic character. Amphiphilic synthetic polymers comprise a wide range of graft, block and star copolymers, while biological examples include nucleic acids, proteins and lipopolysaccharides.

\section{Association and segregation}

Because of the low entropic driving force of mixing, polymer-polymer-solvent systems have a strong tendency to segregative phase separation, i.e., two solution phases enriched in either polymer result. Amphiphiles show a strong tendency to self-assemble into micelles and other structures. Since these have a high effective molecular weight, segregation also becomes a typical feature of mixed polymer-surfactant solutions [4].

The conditions for segregation are entirely different if one of the cosolutes, polymer or surfactant, is ionic. Then the large entropic contribution from the counterions becomes a strong driving force for mixing.

An appreciable attractive interaction between polymer and surfactant will modify the situation and two important phenomena are

1. The binding of surfactant to the polymer.

2. An associative phase separation.

Piculell et al. [5] have identified three distinctly different types of surfactant binding to polymers 
1. An ionic surfactant associates with an oppositely charged polymer.

2. An ionic surfactant shows a strong tendency to associate to a slightly hydrophobic, or a weakly polar, polymer.

3. All surfactants associate to polymers with strongly hydrophobic parts, for example, hydrophobicallymodified water-soluble polymers.

In cases 1 and 2 the interactions are best described in terms of a lowering of the critical micelle concentration (cmc) of the surfactant and in case 3 in terms of the formation of mixed micelles between polymer and surfactant.

\section{Typical phase diagrams}

An aqueous mixture of a rather hydrophobic polymer and a nonionic surfactant can phase-separate into one dilute solution and one concentrated isotropic phase. As illustrated in Fig. 1, the extent of phase separation increases with increasing temperature; this is due to the decreased polarity of both surfactant and polymer at higher temperatures.

A very strong tendency for associative phase separation is displayed by polyelectrolytes mixed with oppositely charged surfactants [7-13]. Here a correct representation of the phase diagram is not possible in the conventional triangular diagram, but a three-dimensional representation, representing the complete four-

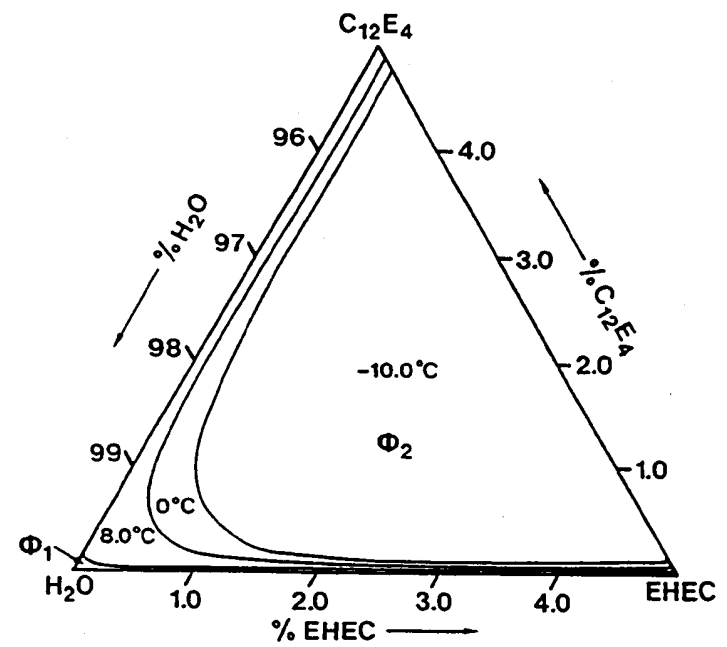

Fig. 1 Hydrophobic interactions between a surfactant and a polymer may induce an associative phase separation; typically this increases with increasing temperature. The phenomenon is exemplified by the aqueous mixture of an oxyethylene nonionic surfactant, $\mathrm{C}_{12} \mathrm{E}_{4}$, and a nonionic polymer, ethyl(hydroxyethyl) cellulose. $\Phi_{1}$ refers to an isotropic one-phase region and $\Phi_{2}$ to an isotropic two-phase region. From Ref. [6] component system, is required. In Fig. 2 the typical behavior is given in the illustrative three-dimensional pyramid diagram. Water is placed at the top and the four sides of the pyramid base are assigned to the four ionic components of the system, with ions of the same charge located at opposite sides. As can be seen, there are two two-phase regions. In the low-salt one there is an associative phase separation, i.e., the surfactant and the polyelectrolyte form a concentrated phase in equilibrium with a very dilute solution. At high salt concentrations there is a segregative phase separation, i.e., there is one surfactant-rich and one polymer-rich solution. At intermediate salt concentrations a homogenous solution is formed. The pyramid representation certainly contains more information than the normally used triangular representation, in which one dimension is omitted, but due to the difficulties to represent the pyramids, triangular phase diagrams are to be preferred in many cases.

\section{DNA and cationic surfactants phase-separate associatively}

The phase diagrams of DNA in combination with different cationic surfactants have been investigated. As illustrated in Fig. 3, there is, as for the analogous

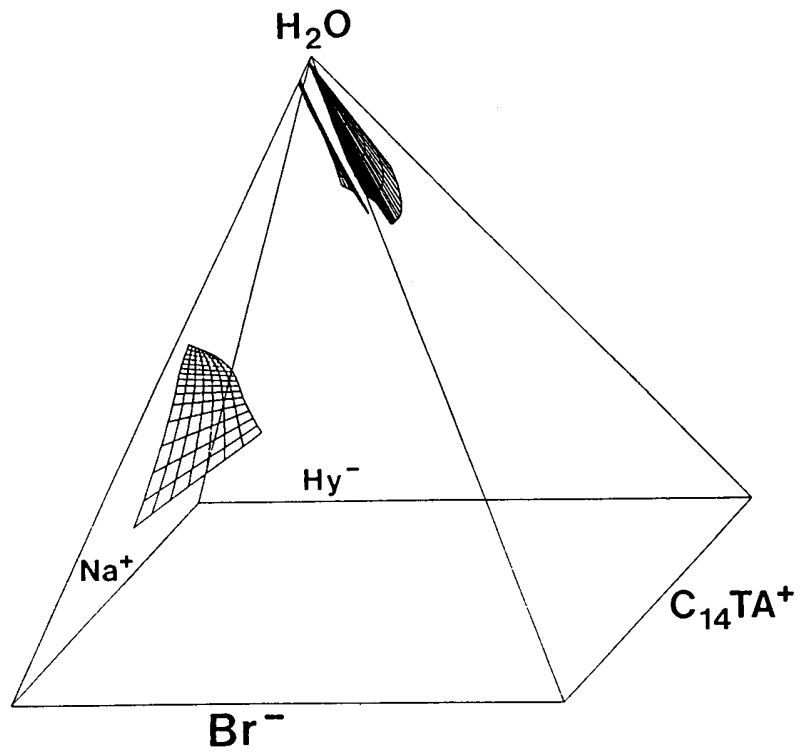

Fig. 2 A polyelectrolyte and an oppositely charged surfactant typically phase-separate associatively at low electrolyte concentrations and segregatively in the presence of high salt contents; at intermediate electrolyte concentrations, there is miscibility. The "sail" hanging from the pyramid top corresponds to the two-phase region. The dilute phase is located to the upper right side, and the concentrated phase to the lower side of the "sail". The example shown is hyaluronan, an anionic polysaccharide, and tetradecyltrimethylammonium bromide, a cationic surfactant. From Ref. [14] 
polyelectrolyte-surfactant systems [7], a strong tendency for associative phase separation. For DNA the role of hydrophobic polymer-surfactant interactions has to be further examined.

\section{DNA compaction in the presence of cationic surfactants - an illustration of association}

With fluorescence microscopy it is possible to visualize individual high-molecular-weight DNA molecules [15]. Because of the high charge density, DNA in aqueous solution exhibits a highly extended coil state at low electrolyte concentrations. DNA compaction to a globule results on screening the electrostatics [16].

On addition of cationic surfactant, DNA compaction takes place [17]. At lower surfactant concentrations, there is a region of coexistence of coils and globules (Fig. 4).

Cationic surfactant binding to DNA is strongly cooperative [18] and is best described as a DNA-induced surfactant self-assembly; direct support for this view comes, apart from from structural investigations, from the fact that the "binding isotherms" are shifted for the different surfactant alkyl chain lengths in the same way as the normal $\mathrm{cmc}$.

Surfactant-induced compaction of a high-molecularweight polymer molecule can be seen as a singlemolecular analogy to the associative phase separation. The different parts of the DNA molecule strive in the presence of the surfactant to form a concentrated domain.
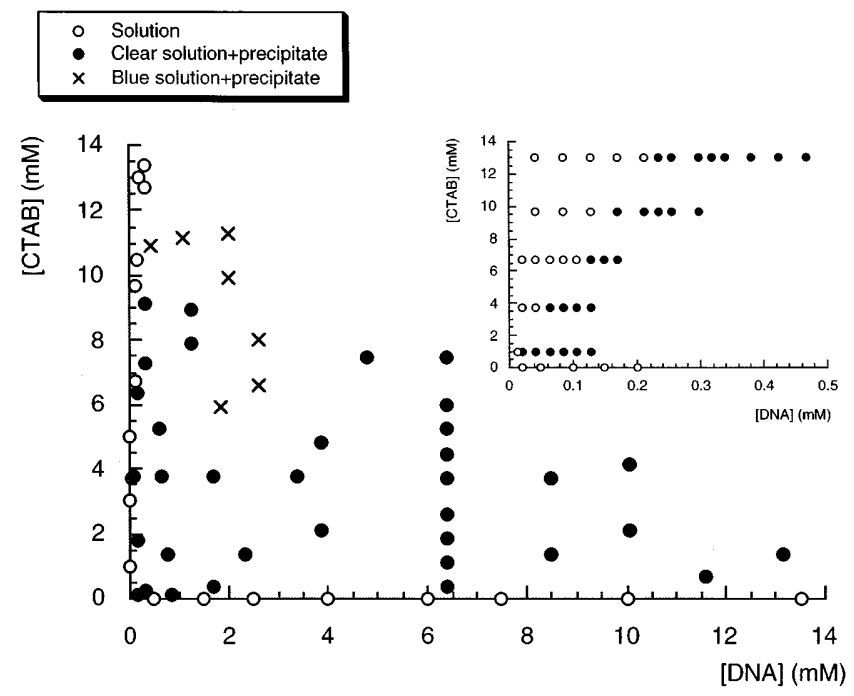

Fig. 3 DNA phase-separates associatively with cationic surfactants. A simplified two-dimensional representation of the preliminary results is given for the hexadecyltrimethylammonium bromide-DNA system (a)

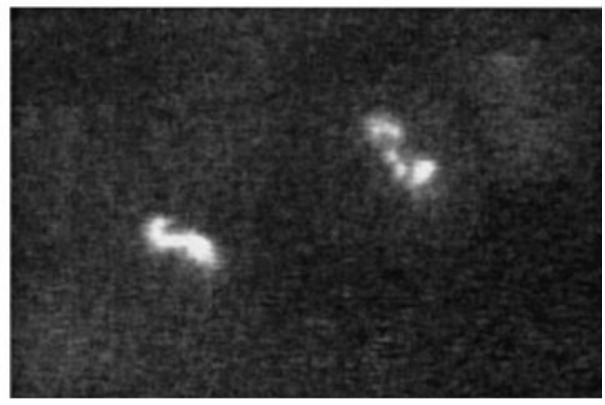

(b)

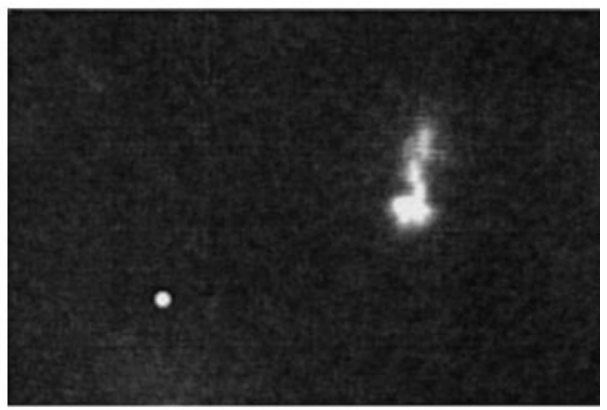

(c)

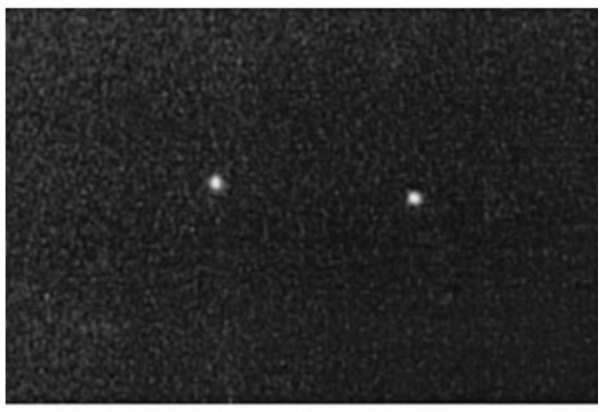

\section{$1 \mathrm{E} \cdot \mathrm{Bm}$}

Fig. 4 Fluorescence microscopy visualizes that the compaction of single DNA is induced by cationic surfactants. As shown for the case of dodecyltrimethylammonium bromide, DNA has an extended coil state at low surfactant concentrations a and a compact globule state at high concentrations $\mathbf{c}$; at intermediate concentrations there is coexistence of coils and globules $\mathbf{b}$

\section{Catanionic vesicles}

Mixed cationic-anionic surfactant systems, referred to as catanionic surfactants [19], offer excellent possibilities to control the charge density of self-assemblies [20]. A particularly interesting feature is the formation of thermodynamically stable vesicles [21]. In the phase diagram, the water-rich part of which is schematically indicated in Fig. 5, there are two regions of vesicle formation. In one the vesicles have a net negative charge, in the other a net positive charge. One of our main 


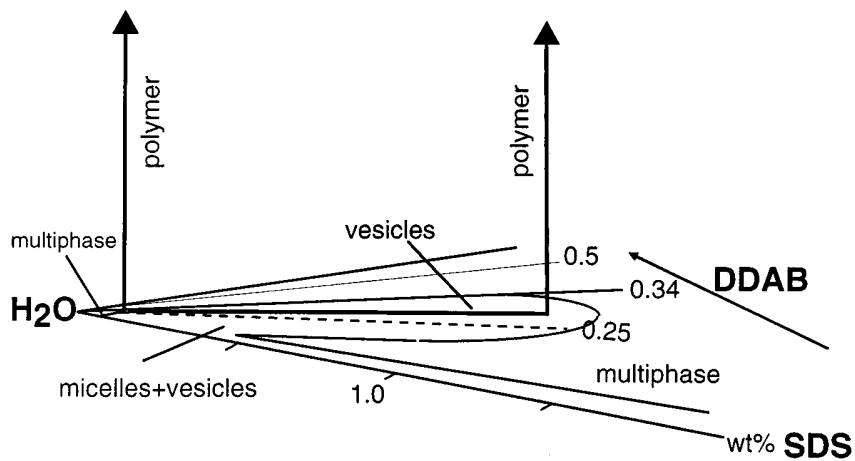

Fig. 5 Schematic representation of the approach used for the study of the phase behavior of polymer-cationic vesicle mixtures; the surfactants studied are didodecyldimethylammonium bromide $(D D A B)$ and sodium dodecylsulfate $(S D S)$. From Ref. [19]

current interests concerns the interaction of catanionic vesicles with polymers [22].

\section{Vesicle-polymer interactions}

The interaction of vesicles with a net negative charge and two cationic cellulose derivatives have been studied: one of these (LM 200, here denoted $\mathrm{HMP}^{+}$) was of the hydrophobically modified type, while the other (JR 400, or $\mathrm{P}^{+}$) was not.

While the phase diagram (Fig. 6) is not yet well investigated in detail, some general features can be singled out:

1. With excess polymer or excess surfactant, a homogenous isotropic solution forms.

2. In a broad intermediate range, associative phase separation results.

3 . There is a close analogy with respect to phase separation with previous studies of polymer-micelle systems.

Rheological investigations with $\mathrm{P}^{+}$show a viscoelastic behavior (Fig. 7). For $\mathrm{HMP}^{+}$the formation of gels is very pronounced.

Cryogenic transmission electron microscopic investigations (Fig. 8) display many notable features, such as the direct visualization of the polymer-induced vesicle association and the formation of faceted vesicles. Similarly DNA was observed to induce an association of vesicles with a net positive charge.

\section{Forthcoming work}

Polymer-surfactant association in aqueous systems is a result of hydrophobic and electrostatic interactions.

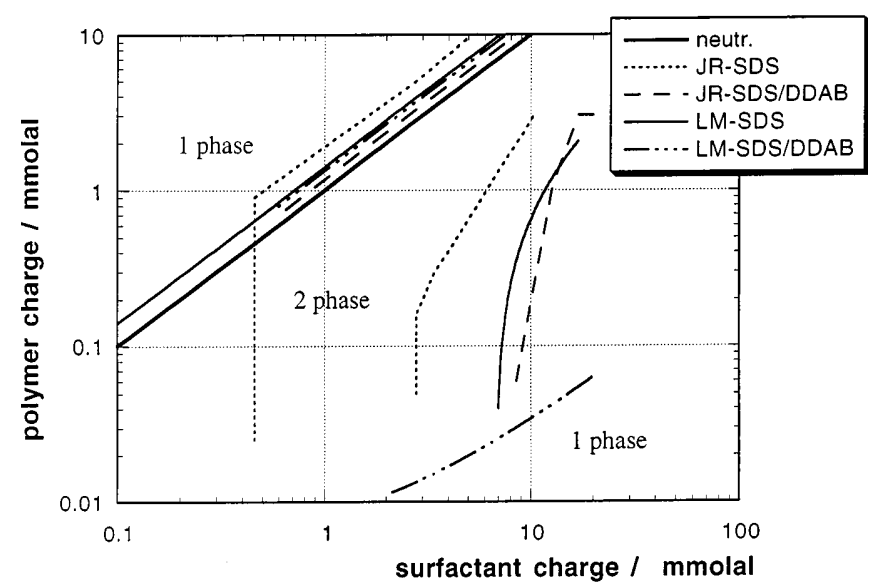

Fig. 6 Comparison of phase maps of mixed polyelectrolyte-surfactant systems show that anionic micellar (SDS) and vesicular (SDSDDAB) systems behave similarly; the phase limits are given as a function of polymer and surfactant charge for two cationic cellulose derivatives, JR 400 and LM 200. Lines at lower surfactant charge: coacervation/precipitation boundaries; lines at higher surfactant charge: redissolution boundaries. For any given system, the region between the two boundaries is a two-phase region. From Ref. [19], data for micellar systems from Refs. [23-24]

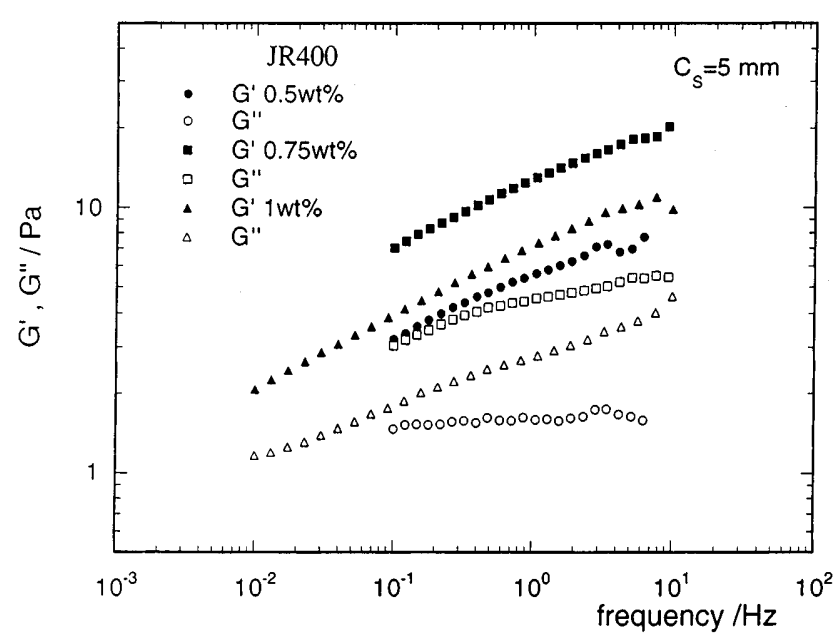

Fig. 7 Mixtures of the cationic polymer JR 400, and catanionic SDSDDAB vesicles have a much higher elastic $\left(G^{\prime}\right)$ than loss modulus $\left(G^{\prime \prime}\right)$ over a wide range of frequencies; data are given for a total surfactant concentration of $5 \mathrm{mM}$ and three different polymer concentrations $(0.50,0.75$, and $1.00 \mathrm{wt} \%)$ at $25^{\circ} \mathrm{C}$. From Ref. [19]

Surfactant self-assembly is a general feature of these systems. If the polymer itself is hydrophobically associating, particularly strong polymer-surfactant association takes place resulting inter alia in viscosifying and gelation.

In this account, we have presented some recent illustrations taken from an ongoing collaboration be- 
Fig. 8 Cryogenic transmission electron microscopy micrographs of aqueous mixtures of a cationic polymer, LM 200, and catanionic vesicles with a net negative charge show a number of interesting features, such as polymer-induced vesicle clustering and faceted vesicles. Scale bar $100 \mathrm{~nm}$. From Ref. [25]
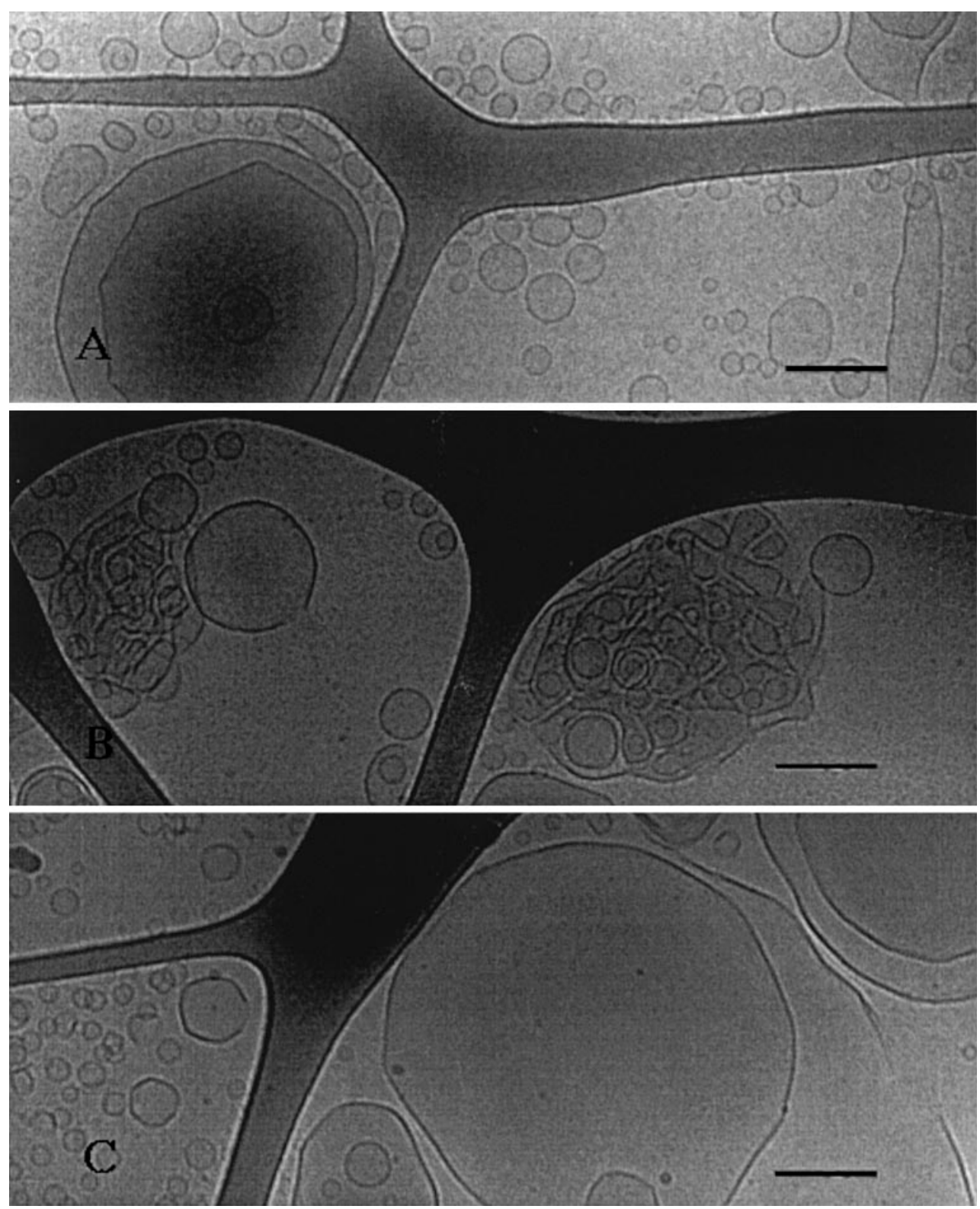

tween our groups in Coimbra and Lund. The work on catanionic surfactant systems is from the $\mathrm{PhD}$ thesis work of Eduardo Marques and an extensive account can be found in his thesis [25]. The work just started on the phase behavior of DNA-surfactant and on DNAcatanionic vesicle interactions is the diploma work of Rita Dias. A full account of this work, with detailed experimental findings and experimental techniques, will be presented later.
Acknowledgements The PhD studies of Eduardo Marques and his stay in Lund were mainly supported by JNICT and Praxis XXI through a research studentship (BD/9295/96) and in part by the Centre of Amphiphilic Polymers (CAP) in Lund. The postdoctoral stay of Sergey Mel'nikov in Lund was supported by the Royal Swedish Academy of Science and the Swedish Natural Sciences Research Council. Rita Dias is an EU Erasmus exchange student. The visiting professorship of Maria Miguel was supported by the CAP. 


\section{References}

1. Jönsson B, Lindman B, Holmberg K Kronberg B (1998) Surfactants and polymers in aqueous solution. Wiley, New York

2. Kwak JCT (ed) (1998) Polymer-surfactant systems. Surfactant science series, vol 77. Dekker, New York

3. Goddard ED, Ananthapadmanabhan KP (eds) (1993) Interactions of surfactants with polymers and proteins. CRC Press, Boca Raton

4. Piculell L, Lindman B (1992) Adv Colloid Interface Sci 41:149

5. Piculell L, Lindman B, Karlström G (1998) In: Kwak JCT (ed) Polymersurfactant systems. Dekker, New York 1998, p 65

6. Zhang $\mathrm{K}$, Karlström G, Lindman B (1994) J Phys Chem 98:4411

7. Lindman B, Thalberg K (1993) In: Goddard ED, Ananthapadmanabhan KP (eds) Interactions of surfactants with polymers and proteins. CRC Press, Boca Raton, p 203

8. Iliopoulos I, Wang TK, Audebert R (1991) Langmuir 7:617
9. Magny B, Iliopoulos I, Audebert R, Piculell L, Lindman B (1992) Prog Colloid Polym Sci 89:118

10. Hansson P, Almgren M (1995) J Phys Chem 99:16684

11. Hansson P, Almgren M (1995) J Phys Chem 99:16694

12. Hansson P, Almgren M (1996) J Phys Chem 100:9038

13. Anthony O, Zana R (1996) Langmuir $12: 1967$

14. Thalberg K, Lindman B (1991) In: Mittal KL, Shah DO (eds) Surfactants in solution, vol 11. Plenum, New York, p 243

15. Bustamante C (1991) Annu Rev Biophys Biophys Chem 20:415

16. Grosberg AYu, Khokhlov AR (1996) Giant molecules. Academic Press, London

17. Mel'nikov SM, Sergeyev VG, Yoshikawa K (1997) In: Pandalai SG (ed) Recent research developments in chemical sciences, vol 1. Transworld Research Network, Trivandrum, India, p 69
18. Hayakawa K, Kwak JCT (1991) In: Rubingh DN, Holland PM (eds) Cationic surfactants: physical chemistry. Dekker, New York, p 189

19. Khan A, Marques E (1997) In: Robb ID (ed) Specialists surfactants. Chapman \& Hall, London, p 37

20. Lindman B, Khan A, Marques E, Miguel MG, Piculell L, Thalberg K (1993) Pure Appl Chem 65:953

21. Kaler EW, Murthy AK, Rodriguez BE, Zasadzinski JA (1989) Science 245: 1371

22. Marques E, Regev O, Khan A, Miguel MG (1999) Langmuir 15:642

23. Guillemet F, Piculell L (1995) J Phys Chem 99:9203

24. Goddard ED, Hannan RB (1977) J Am Oil Chem Soc 54:561

25. Marques E (1998) PhD Thesis. Universidade de Coimbra, Portugal 